\title{
An application of MDLPF models for solar radiation forecasting
}

\author{
Emre Akarslan, Fatih Onur Hocaoglu* \\ Afyon Kocatepe University, ANS Campus Engineering Faculty 03200 Afyonkarahisar, Turkey \\ Solar and Wind Energy Applications and Research Center, ANS Campus 03200 Afyonkarahisar, Turkey
}

\begin{abstract}
Electricity generation from renewable resources is a hot topic due to increasing environmental awareness of people and energy needs. Solar energy is one of the most important clean energy sources. Forecasting of solar radiation is one of the important stages in sizing and managing a PV power plant. Moreover since the smart grid applications are started, accurate forecasting of the energy output of PV system (hence the solar radiation) became a hot topic. In literature there are a huge number of studies tries to find more accurate forecasting models. Among them in this study Multi-Dimensional Linear Prediction Filter Models (MDLPF Models) are studied. To test the performance of MDLPF models, hourly solar radiation data of two different regions (Ankara and Çanakkale) are employed. In forecasting five different MDLPF Models are built. The accuracies of the forecasting results are compared and discussed.
\end{abstract}

Keywords: Solar radiation, forecasting, MDLPF, linear prediction

\section{Introduction}

By the conversion from conventional distribution grids to intelligent one, renewable resources are started to integrate to the grid in an efficient way. Power generation from solar energy is an attractive issue in energy world and there are a lot of studies about this subject. The production of a PV panel is directly related to the insolation at its location and solar radiation varies because of changes in the diurnal and seasonal position of the sun relative to the Earth. To manage a grid supplied by different sources, generations from the plant must be accurately predicted. Therefore solar radiation forecasting is an important topic in energy field and there are a lot of methods to predict solar radiation such as AutoRegressive (AR) [1], Auto-Regressive and Moving Average (ARMA) [2], Auto-Regressive and Integrated Moving Average [3], Markov Chains [4], Fuzzy Logic [5], Wavelet network [6], Adaptive Neuro-Fuzzy Inference System (ANFIS) [7], ARMA/ANN [8] and Artificial Neural Networks (ANNs) [9] etc. Ref. [10] applied time series analysis to forecast next hour solar irradiance including cloud cover effects in their study. They proposed three forecasting methods using different types of meteorological data as input parameters, namely, Global Horizontal Irradiance (GHI), Diffuse Horizontal Irradiance (DHI), Direct Normal Irradiance (DNI) and cloud cover. Ref. [11] proposed a neural network based procedure to predict hourly solar radiation values. In this procedure not only the hourly measured solar radiations but also the hourly calculated extraterrestrial radiation data are employed. They tested their procedure on hourly measured yearly global solar radiation data obtained from Afyonkarahisar region and showed the effectiveness of their method.

Ref. [12] used 2-D linear filters to forecast the hourly solar radiation data. They proposed a novel representation of solar radiation data. First, the annual solar radiation data are transformed into a 2-D image. The rows and columns of the image correspond to days and hours, respectively. Then, different

\footnotetext{
* Manuscript received June 23, 2015; revised September 15, 2015.

Corresponding author. Tel.: +902722281423; E-mail address: e.akarslan@gmail.com.

doi: $10.12720 /$ sgce.4.4.299-304
} 
types of linear filters with various filter-tap configurations are applied to the image, and the optimal filter coefficients are calculated. Ref. [13] proposed a novel hybrid method to forecast hourly solar radiation. This method use the linear prediction filters and an empiric model based on extraterrestrial radiation. In case a sunny day condition the alteration of the data will smoother therefore linear prediction based approach is used otherwise, an empirical model is preferred. Ref. [14] proposed M-D (Multi-Dimensional) linear prediction filter models to forecast hourly solar irradiance values. The novelty of these models is that they utilize not only the solar irradiance image but also different images that correlate with solar irradiance data. These filters are employed both to link the images with each other and to predict solar irradiance data. They provided better results at a range 1\%-30\% with different M-D models compared with 2-D filters.

In this study, MDLPF approach applied on solar radiation data belong to two regions (Ankara, Çanakkale) in Turkey. Extraterrestrial radiation and future values of extraterrestrial radiations are used for additional information in the forecasting environment beside recorded solar radiation values. First, solar radiation, extraterrestrial radiation and future values of extraterrestrial radiation time series are converted to the images. Then, MDLPF templates are built and applied for forecasting. There parameters are considered in formation of MDLPF. These parameters are the data (solar and extraterrestrial radiations) belonging to the measured values of; this hour, one hour before and one day before, respectively.

The organization of the paper is as follows. The description and representation of data used for this study are described in Section 2. The M-D linear prediction filter approach is explained in Section 3. The experimental results are illustrated in Section 4. Finally, conclusions are explained in Section 5.
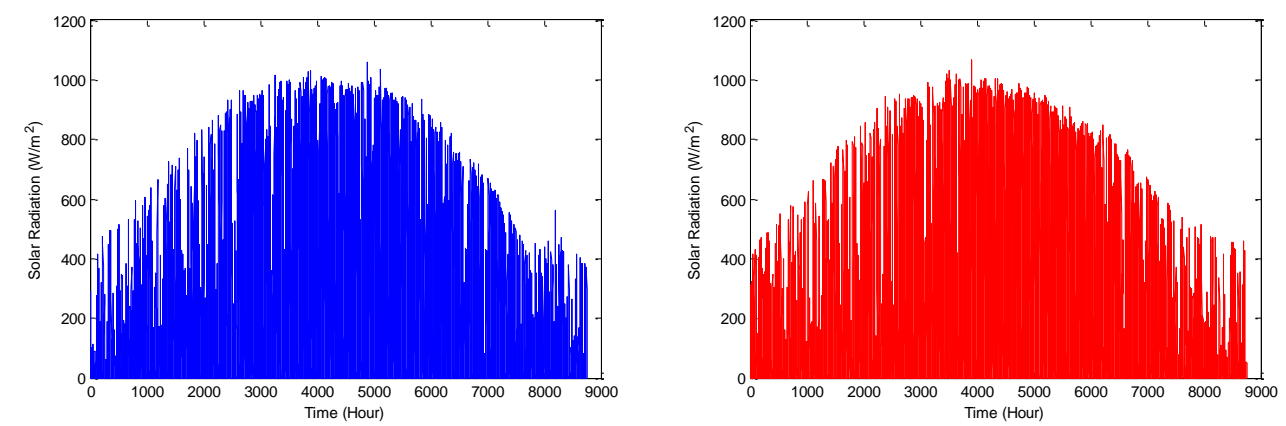

Fig. 1. The hourly solar radiations for Ankara (left) and Çanakkale (right).

\section{Description and Representation of Data Used}

In this study, one year hourly solar radiation data measured from Ankara and Çanakkale regions of Turkey are used. These data are taken from the Turkish State Meteorological Service (DMI). The variation in the solar irradiance value over a 1-year period from 2013 for Ankara and Çanakkale are shown in Fig. 1. Ankara and Çanakkale regions do not have a good insolation characteristic. Ankara has better potential then Çanakkale. The extraterrestrial irradiance is the intensity of the sun at the top of the Earth's atmosphere and is calculated using solar geometry for the region. It varies throughout the year because of the Earth's elliptical orbit, which results in an Earth-Sun varying distance during the year in a predictable way [14]. To account for the eccentricity of the Earth's orbit around the sun, an eccentricity correction factor is used, as in equation (1):

$$
I_{0}=1367.7 x\left(1+0.033 \cos \left(\frac{360 x n}{365}\right)\right)
$$

where $n$ is the day of the year. For January $1, n$ is 1 , and for December $31, n$ is 365 . Spencer (1971) created a more detailed model through the Fourier series, as in equation (2): 


$$
\begin{aligned}
& I_{0}=I_{s c}[1.00011+0.034221 x \cos (\mathrm{X})+0.00128 x \sin (\mathrm{X})-0.000719 x \cos (2 \mathrm{X}) \\
& +0.000077 x \sin (2 \mathrm{X})]
\end{aligned}
$$

where $I_{s c}$ is the solar constant and is equal to $1366.1 \mathrm{~W} / \mathrm{m}^{2}$. The NREL's SOLPOS algorithm also calculates extraterrestrial radiation. The three models vary slightly from one another, but they have the same general shape for calculating extraterrestrial radiation [15]. In this study, hourly extraterrestrial global solar radiations are calculated using the MDIC SOLPOS Calculator, which is available from the NREL website (http://www.nrel.gov/).

After the extraterrestrial radiation and solar radiation time series are obtained, the data are converted into a 2-D matrix. Hocaoglu et al. (2008) first proposed this 2-D representation of solar irradiance data. The 2-D image obtained from the solar irradiance data provides the tool for the image processing techniques on solar data. In this study solar radiation data, extraterrestrial radiation data and derivatives of these images are used. To predict solar irradiance, these images are linked to different designs of optimal coefficient linear prediction filters. A linkage process is applied to these images. This process involves connecting different images that correspond to different parameters. In the linkage phase, the same pixel indices of different images are connected to each other by filter taps to calculate the prediction values (pixels) of the solar irradiance image. In this way, a combination of different images in a Multi-layered form is obtained. Next, the pixel values of these data are evaluated for the prediction model. This technique enables to represent different information compactly and in the same pattern. The combination of different images in the Multi-layered form is necessary when using 3-D filter templates. These templates are 3-D and scan the image in a format 2-D. Therefore, they utilize the information on each layer of the Multi-layered form to predict solar irradiance [15].

\section{MDLPF Approach}

The MDLPF provide to utilize information from different parameters correlated with solar radiation data. In this approach, the MDLPFs are applied to the image, which consists of the solar radiation and correlated data; the optimal filter coefficients are determined, consecutively. The filter template (Eq. (3)) which uses the actual value, one hour before and one day before data to predict one hour later solar radiation value, is used. The prediction pixel is determined using the past pixels with Eq. (4).

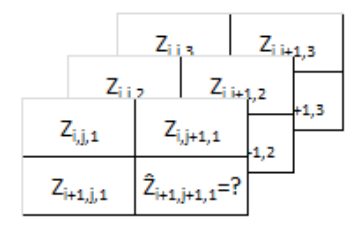

$$
\check{Z}_{i+1, j+1,1}=Z_{i, j, 1} \cdot a_{1}+Z_{i, j+1,1} \cdot a_{2}+Z_{i+1, j, 1} \cdot a_{3}+Z_{i, j, 2} \cdot a_{4}+Z_{i, j+1,2} \cdot a_{5}+Z_{i+1, j, 2} \cdot a_{6}+Z_{i, j, 3} \cdot a_{7}+Z_{i, j+1,3} \cdot a_{8}+Z_{i+1, j, 3} \cdot a_{9}(4)
$$

$i, j$ and $Z_{i, j}$ are identified as the row and column number of the pixel and the pixel value, respectively. In MD solar data, $i$ denotes the days, $j$ denotes the hours and $Z_{i, j}$ denotes the solar radiation or correlated data at day $i$ and hour $j$. A prediction is made for all possible $(i, j)$ coordinates using past samples. The combination coefficients are optimized using a minimum squared error between the estimation and the real value. The error at a particular coordinate $(i, j)$ is calculated using Eq. (5) [14].

$$
\varepsilon_{i+1, j+1}=\check{Z}_{i+1, j+1}-Z_{i+1, j+1}
$$

The energy of the total prediction error is, therefore, calculated as: 
$\varepsilon=\sum_{i=2}^{m} \sum_{j=2}^{n} \varepsilon_{i, j}^{2}$

where $m$ and $n$ identify the size of the image. The filter coefficients, which minimize this function, can be calculated using Eq. (7):

$$
\frac{\partial \varepsilon}{\partial a_{1}}=\frac{\partial \varepsilon}{\partial a_{2}}=\frac{\partial \varepsilon}{\partial a_{3}}=\frac{\partial \varepsilon}{\partial a_{4}}=\frac{\partial \varepsilon}{\partial a_{5}}=\frac{\partial \varepsilon}{\partial a_{6}}=\frac{\partial \varepsilon}{\partial a_{7}}=\frac{\partial \varepsilon}{\partial a_{8}}=\frac{\partial \varepsilon}{\partial a_{9}}=0
$$

The solution to Eq. (7) yields the following equation:

$$
\left[\begin{array}{lllllllll}
r_{11} & r_{12} & r_{13} & r_{14} & r_{15} & r_{16} & r_{17} & r_{18} & r_{19} \\
r_{21} & r_{22} & r_{23} & r_{24} & r_{25} & r_{26} & r_{27} & r_{28} & r_{29} \\
r_{31} & r_{32} & r_{33} & r_{34} & r_{35} & r_{36} & r_{37} & r_{38} & r_{39} \\
r_{41} & r_{42} & r_{43} & r_{44} & r_{45} & r_{46} & r_{47} & r_{48} & r_{49} \\
r_{51} & r_{52} & r_{53} & r_{54} & r_{55} & r_{56} & r_{57} & r_{58} & r_{59} \\
r_{61} & r_{62} & r_{63} & r_{64} & r_{65} & r_{66} & r_{67} & r_{68} & r_{69} \\
r_{71} & r_{72} & r_{73} & r_{74} & r_{75} & r_{76} & r_{77} & r_{78} & r_{79} \\
r_{81} & r_{82} & r_{83} & r_{84} & r_{85} & r_{86} & r_{87} & r_{88} & r_{89} \\
r_{91} & r_{92} & r_{93} & r_{94} & r_{95} & r_{96} & r_{97} & r_{98} & r_{99}
\end{array}\right]\left[\begin{array}{l}
a_{1} \\
a_{2} \\
a_{3} \\
a_{4} \\
a_{5} \\
a_{6} \\
a_{7} \\
a_{8} \\
a_{9}
\end{array}\right]=\left[\begin{array}{c}
r_{1} \\
r_{2} \\
r_{3} \\
r_{4} \\
r_{5} \\
r_{6} \\
r_{7} \\
r_{8} \\
r_{9}
\end{array}\right]
$$

where $r_{i j}$ is the correlation value between the past values, $a_{i}$ is the linear filter coefficients and $r_{k}$ is the correlation between the past and predicted pixel. This matrix equation can be expressed as R.a=r, and the optimal filter coefficients can be found thus [14]:

$$
a=R^{-1} . r
$$

\section{Experimental Results}

In this study, five different models with one filter template are formed to forecast hourly solar radiation data. Model 1 uses only past sample of solar radiation data and this is a sample for 2-D linear prediction filters. Model 2 and Model 3 use the past sample of extraterrestrial radiation and future value of extraterrestrial radiation with solar radiation, respectively. Model 4 and Model 5 use the derivatives of extraterrestrial radiation and future value of extraterrestrial radiation. The RMSE criterion is selected as a performance measure of the models. The RMSE is a commonly used measure of the differences between the values extracted by a forecasting model or an estimator and the observed values. The value of the RMSE provides information on the short term performance [16]. Model 1-5 with filter template given in Eq. 3 are applied to solar data belong to Ankara and Çanakkale regions of Turkey in time period 1st January 2013- 31th December 2013. Results are given in Table 1.

Table 1. Prediction performances of each model

\begin{tabular}{|l|l|l|l|}
\hline \multirow{2}{*}{ Model } & \multirow{2}{*}{ Data Used in Model } & \multicolumn{2}{|l|}{ RMSE $\left(\mathbf{W} / \mathbf{m}^{2}\right)$} \\
\cline { 3 - 4 } & & Ankara & Çanakkale \\
\hline Model-1 & Solar Radiation & 74.9968 & 64.8303 \\
\hline Model-2 & Extraterrestrial-Solar Radiation & 61.9955 & 53.5594 \\
\hline Model-3 & Futurre Extraterrestrial-Solar Radiation & 64.5341 & 56.8679 \\
\hline Model-4 & $\begin{array}{l}\text { First order derivative of extraterrestrial } \\
\text { radiation-Solar radiation }\end{array}$ & 65.5528 & 53.7397 \\
\hline Model-5 & $\begin{array}{l}\text { First order derivative of future } \\
\text { extraterrestrial radiation-Solar radiation }\end{array}$ & 62.6642 & 54.2143 \\
\hline
\end{tabular}

As seen in Table 1, all models which uses M-D filter approach (Model 2-5) give better results than 2-D 
one (Model 1). This means to provide additional information improve the prediction accuracy and the MD filter approach provide the possibility for use of different parameters. The best results are obtained with Model-2 which uses extraterrestrial and solar radiation to predict solar radiation. Extraterrestrial radiations are theoretical limit of solar radiation for any hour and provide important information for it.

The correlation between the actual and predicted values for Çanakkale with Model 2 is shown in Fig. 2. It is clear from Fig. 2 that prediction results are closely matching the actual data along the diagonal axis. Therefore, the slope of the linear fit for Fig. 2 is close to 45 degrees, and the scatter is narrow along the diagonal axis. These observations indicate a high forecasting success for the model. The mesh plot of the prediction error for this model is presented in Fig. 3. The error pixels are almost uncorrelated with each other. Therefore, the model works with very good accuracy.

It is obtained that in case of first order derivative of extraterrestrial radiation is used, the accuracy of the prediction can be improved, however, not as much as extraterrestrial radiation. Using first order derivative of future extraterrestrial radiation instead of itself, gives better prediction results. The future variation in extraterrestrial radiation carries important information about the future variation in solar radiation.

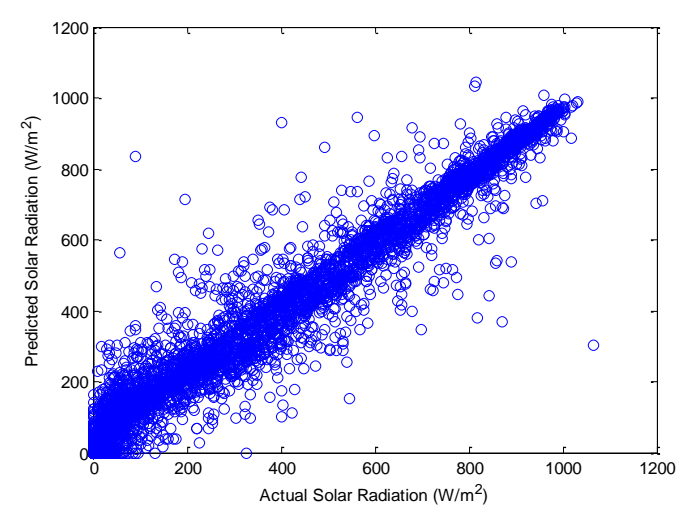

Fig. 2. Correlation between the actual and predicted solar radiation for model 2 (Çanakkale).

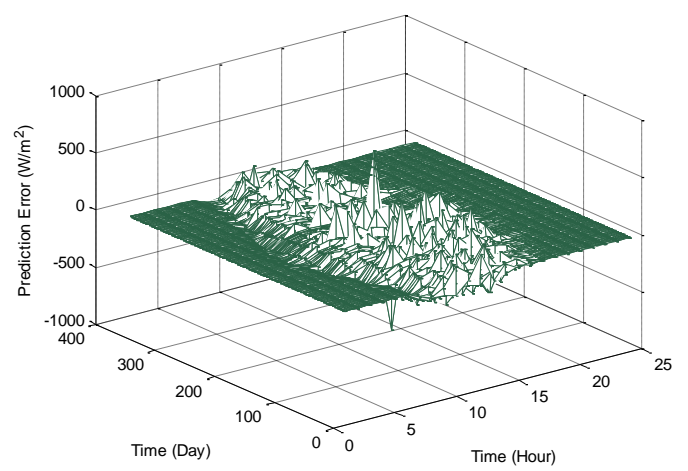

Fig. 3. Mesh plot of the prediction error for model 2 (Çanakkale).

\section{Conclusion}

In this work, the MDLPF approach is used for hourly solar radiation forecasting of two cities of Turkey. This technique evaluates annual hourly solar radiations, extraterrestrials and their derivatives over time as multi-dimensional images. These images are linked with each other with the help of the M-D filters. These filters scan data pattern. By this approach pixel values corresponding to next hour's solar radiation data can be predicted. The performance of 5 different models on the Ankara and Çanakkale data 
is tested. It is obtained that MDLPFs outperform to 2-D linear prediction filters. It is finally obtained that the models can be used for any data.

\section{Acknowledgements}

This study is financed by Afyon Kocatepe University BAP (THE SCIENTIFIC RESEARCH PROJECTS) Coordination Unit by the project number 15.HIZ.DES.68.

\section{References}

[1] Bacher P, Madsen H, Nielsen HA. Online short-term solar power forecasting. Solar Energy. 2009; 83:1772-1783.

[2] López LM, Cardona MS. Cardona. Multiplicative ARMA models to generate hourly series of global irradiation. Solar Energy. 1998; 63(5):283-291.

[3] Box GEP, Jenkins GM, Reinsel GC. Time Series Analysis: Forecasting and Control. 4 $^{\text {th }}$ ed. Wiley; 2008.

[4] Amato U, Andretta A, Bartolli B, Coluzzi B, Cuomo V, Fontana F. Markov process and Fourier analysis as a tool to describe and simulate solar irradiation. Solar Energy. 1996; 37:197-201.

[5] Boata RST, Gravila P. Functional fuzzy approach for forecasting daily global solar irradiation. Atmospheric Research. 2012; 112:79-88.

[6] Mellit A, Benghanem M, Kalogirou SA. An adaptive wavelet-network model for forecasting daily total solar radiation. Applied Energy. 2005; 83:705-722.

[7] Moghaddamnia A, Remesan R, Kashani MH, Mohammadi M, Han D, Piri J. Comparison of LLR, MLP, Elman, NNARX and ANFIS modelsdwith a case study in solar radiation estimation. Atmospheric Solar-Terrestrial Phys. 2009; 71:975-982.

[8] Voyant C, Musseli M, Paolia C, Nivet ML. Numerical Weather Prediction (NWP) and hybrid ARMA/ANN model to predict global radiation. Energy. 2012; 39:341-355.

[9] Notton G, Paoli C, Ivanova L, Vasileva S, Nivet ML. Neural network approach to estimate 10-min solar global irradiation values on tilted planes. Renewable Energy. 2013; 50:576-584.

[10] Dazhi Y, Jirutitijaroen P, Walsh WM. Hourly solar irradiance time series forecasting using cloud cover index. Solar Energy. 2012; 86:3531-3543.

[11] Serttas F, Hocaoglu FO, Akarslan E. A new NN based procedure for hourly solar radiation forecasting. Presented at: 2nd International Conference on Water, Energy and Environment, 2013.

[12] Hocaoglu FO, Gerek ON, Kurban M. Hourly solar radiation forecasting using optimal coefficient 2-D linear filters and feedforward neural networks. Solar Energy. 2008; 82:714-726.

[13] Akarslan E, Hocaoglu FO. A novel hybrid model for hourly solar radiation forecasting. In: Proc. 7th International Ege Energy Symposium \& Exhibition, 2014:44-52.

[14] Akarslan E, Hocaoglu FO, Edizkan R. A novel M-D (multi-dimensional) linear prediction filter approach for hourly solar radiation forecasting. Energy. 2014; 73:978-986.

[15] Reno MJ, Hansen CW, Stein JS. Global horizontal irradiance clear sky models: implementation and analysis. Sandia Report, Sandia National Laboratories, 2012.

[16] Nastos PT, Paliatsos AG, Koukouletsos KV, Larissi IK, Moustris KP. Artificial neural networks modeling for forecasting the maximum daily total precipitation at Athens, Greece. Atmospheric Research. 2013; 144:141-150. 